\title{
SYNTHESIS OF PYRAZOLO [3',4':4,5] PYRIMIDO [2,3-c][1,4] \\ BENZOXAZINES: A NEW HETEROCYCLIC RING SYSTEM
}

\author{
P.S.N. Reddy and Pragati Reddy \\ Department of Chemistry, Osmania University, Hyderabad-500 007, India, \\ E-mail: psnreddyou@yahoo.com
}

and

\section{G. Jagath Reddy * and K. Srinivasa Rao}

R \& D Laboratories, Dr. Jagath Reddy's Heterocyclics, 81, S.V.Co-op Industrial Estate, Balanagar, Hyderabad - 500 037, India. E-maii-jagathreddy@usa.net; Fax \# 91-40-23773487.

\section{Abstract}

A series of 4-oxo-pyrazolo[ $\left.3^{\prime}, 4^{\prime}: 4,5\right]$ pyrimido[2,3-c][1,4]benzoxazines(4a-l) have been prepared by cyclocondensation of 1,4-benzoxazinones (1) and with 5aminopyrazole-4-carboxylic acids(3a-c) in a single step.

\section{Introduction}

A number of 1,4-benzoxazines have been reported to possess pharmacological properties such as antimicrobial, cardiotonic, adrenoceptor and antiparasitic activities ${ }^{1-4}$. These observations created interest in the synthesis of 1,4-benzoxazine fused heterocycles like imidazo- ${ }^{5}$, triazolo- $-^{6}$, triazino- ${ }^{7}$ and pyridobenzoxazines ${ }^{8}$ with a view to obtain new systems with enhanced biological profile. Furthermore, a wide variety of pyrazoles and fused pyrazoles exhibited analgesic, antipyretic and antiinflammatory activities. Also, several pyrazolopyrimidines are known as potential bactericidal, fungicidal and phosphodiesterase inhibiting agents ${ }^{10}$. In view of this and in continuation of our work on new heterocyclic systems ${ }^{11}$, we now report the synthesis of another new tricyclic system namely pyrazolopyrimidobenzoxazines.<smiles>[R]c1ccc(-n2ncc(C(=O)O)c2N)cc1-n1ncc2c(=O)n3c(nc21)OC([R3])C(C)C3</smiles>

a) $\mathrm{POCl}_{3} /$ triethylamine, b) Dichloroethane

S C H E M E - 1 


\section{Results and Discussion}

The starting materials, 2 and 6 substituted benzoxazinones (1) required in the present work were prepared by the condensation of 4-substituted-2-aminophenols with suitably substituted $\alpha$-haloacyllhalides in methyl isobutyl ketone in presence of aqueous sodium bicarbonate according to the reported method ${ }^{12}$. 1-Aryl-5-aminopyrazole-4carboxylic acids were prepared by hydrolysis of the corresponding esters. These esters were prepared from reaction of substituted phenylhydrazines with 2-cyano-3-ethoxy propenate according to the reported method ${ }^{13}$.

Our previous work on tetrazolo $-{ }^{14}$ and quinazolinobenzoxazines ${ }^{15}$ has exemplified the high reactivity of iminochlorides obtained from benzoxazinones towards nucleophiles. Based on this lead, we have now developed a convenient and facile method of converting 1 in a single step into pyrazolopyrimidobenzoxazines (4) via their iminochlorides (2). Thus 1 on reaction with phosphorousoxychloride in dichloroethane at $5^{\circ}$ gave the corresponding imino chlorides (2) which underwent smooth cyclocondensation when treated with 5-aminopyrazole-4-carboxylic acids ${ }^{13}$ (3) to give the hitherto unreported new tricyclic ring system namely, pyrazolo[3', $\left.4^{\prime}: 4,5\right]$ pyrimido[2,3-c][1,4]benzoxazines (4a) in 40-50\% yields (Scheme -1 ). The reaction proceeds, presumably via imidoylchloride formation, followed by in situ condensation with 5-aminopyrazole-4-carboxylic acid (3) and spontaneous cyclodehydration. The structures of products $4 a-1$ were confirmed by microanalyses, $I R$, ${ }^{1} \mathrm{H}-\mathrm{NMR}$ and Mass spectra.

TABLE 1- PHYSICAL DATA OF PYRAZOLO[3',4':4,5]PYRIMIDO[2,3-c][1,4] BENZOXAZINES (4)

\begin{tabular}{|c|c|c|c|c|c|}
\hline Compound & $\mathrm{R}_{1}$ & $\mathbf{R}_{2}$ & $\mathbf{R}_{\mathbf{3}}$ & \multicolumn{2}{|c|}{$\begin{array}{c}4 \\
\text { Yield\% m.p }{ }^{\circ} \mathrm{C} \\
\end{array}$} \\
\hline $\mathbf{a}$ & $\mathrm{H}$ & $\mathrm{H}$ & $\mathrm{H}$ & 47 & $169^{\circ}$ \\
\hline b & $\mathrm{H}$ & $\mathrm{Cl}$ & $\mathrm{H}$ & 49 & $198^{\circ}$ \\
\hline c & $\mathrm{H}$ & $\mathrm{CH}_{3}$ & $\mathrm{H}$ & 45 & $208^{\circ}$ \\
\hline d & $\mathrm{Cl}$ & $\mathrm{H}$ & $\mathrm{H}$ & 52 & $187^{\circ}$ \\
\hline e & $F$ & $\mathrm{H}$ & $\mathrm{H}$ & 54 & $171^{\circ}$ \\
\hline f & $\mathrm{F}$ & $\mathrm{Cl}$ & $\mathrm{H}$ & 51 & $218^{\circ}$ \\
\hline g & $\mathrm{CH}_{3}$ & $\mathrm{H}$ & $\mathrm{H}$ & 42 & $224^{\circ}$ \\
\hline h & $\mathrm{CH}_{3}$ & $\mathrm{Cl}$ & $\mathrm{H}$ & 46 & $244^{\circ}$ \\
\hline $\mathrm{i}$ & $\mathrm{H}$ & $\mathrm{H}$ & $\mathrm{CH}_{3}$ & 43 & $192^{\circ}$ \\
\hline $\mathrm{j}$ & $\mathrm{Cl}$ & $\mathrm{H}$ & $\mathrm{CH}_{3}$ & 48 & $181^{\circ}$ \\
\hline $\mathbf{k}$ & $\mathrm{CH}_{3}$ & $\mathrm{H}$ & $\mathrm{CH}_{3}$ & 47 & $205^{\circ}$ \\
\hline 1 & $\mathrm{COCH}_{3}$ & $\mathrm{H}$ & $\mathrm{H}$ & 38 & $180^{\circ}$ \\
\hline
\end{tabular}


In the ${ }^{1} \mathrm{H}-\mathrm{NMR}$ spectra, the compounds exhibited signals around $\delta 5.0$ for $-\mathrm{OCH}_{2}$ group of benzoxazine ring, a singlet around $\delta 8.3$ for pyrazole proton and the $\mathrm{C}_{6}$ proton appeared around $\delta 8.6$ due to peri effect apart from other aromatic protons. Mass spectral fragmentation of compound $4 \mathrm{a}$ exhibited a prominent molecular ion peak at $\mathrm{m} / \mathrm{z}$ $317(\mathrm{~m}+1)$ along with other fragment ions at $\mathrm{m} / \mathrm{z} 288$ (loss of $\mathrm{CO}$ ), 196, 143 and 77 . The structures of all the compounds reported in Table -1 were based on their IR, ${ }^{1} \mathrm{H}-\mathrm{NMR}$ spectra and correct elemental analyses.

Thus the present method offers a very convenient mild and rapid one step conversion of lactam 1 to the pyrazolo $\left[3^{\prime}, 4^{\prime}: 4,5\right]$ pyrimido[2,3-c][1,4]benzoxazine tricyclic system.

\section{$\underline{\text { Experimental }}$}

Meting points were determined in open capillaries and are uncorrected. IR spectra was recorded in $\mathrm{KBr}$ pellets. ${ }^{1} \mathrm{H}-\mathrm{NMR}$ spectra on a varous $200 \mathrm{MHz}$ instrument with TMS as internal standard and chemical shifts are expressed in $\delta \mathrm{ppm}$ and Mass spectra on a Hewelett Packard Mass Spectrometer operating at 70ev. All the compounds were purified by column chromatography using silica gel.

\section{4,11-Dihydropyrazolo[3',4':4,5]pyrimido[2,3-c][1,4]-benzoxazine-4-one: 4a}

To a mixture of 3-oxo-3,4-dihydro-2H-1,4-benzoxazine (1a, $1.49 \mathrm{~g}, 0.01 \mathrm{~mol})$ in 1,2-dichloroethane $(25 \mathrm{ml})$, phosphorousoxychloride $(2.3 \mathrm{~g}, 0.015 \mathrm{~mol})$ is added dropwrise and the mixture is stirred at room temperature for 30 minutes. Triethylamine $(1.5 \mathrm{~g}, 0.015 \mathrm{~mol})$ is added with stirring at $0-5^{\circ}$, followed by addition of 5aminopyrazole-4-carboxylicacid $\left(3, \mathrm{R}_{2}=\mathrm{H}, 2.03 \mathrm{~g}, 0.01 \mathrm{~mol}\right)$, maintaining the temperature at $5-10^{\circ}$. The mixture is stirred at room temperature for $1 \mathrm{hr}$ and refluxed for $4 \mathrm{hrs}$ on steam bath. The progress of the reaction is followed by TLC. At the end of the reaction, cold water $(50 \mathrm{ml})$ is added to the reaction mixture and the organic layer is separated, washed with water, $10 \%$ aqueous sodiumbicarbonate, water, dried and concentrated to give crude $4 \mathrm{a}$. The crude products were purified by column chromatography using silica gel and eluted with a mixture of hexane and ethylacetate (4:1) to give pure $4 \mathrm{a}$ as crystalline solid $(1.48 \mathrm{~g}, 47 \%) \mathrm{mp}: 168^{\circ}$; IR (KBR): $1710 \mathrm{~cm}^{-1} \mathrm{~ms}$ (70ev) $\mathrm{m} / \mathrm{z}(\%) 317(\mathrm{M}+1):{ }^{1} \mathrm{H}-\mathrm{NMR}\left(\mathrm{CDCl}_{3}\right): \delta 5.0\left(\mathrm{~s}, 2 \mathrm{H}, \mathrm{OCH}_{2}\right), 7.1-7.6(\mathrm{~m}, 6 \mathrm{H}, \mathrm{ArH})$ $8.1(\mathrm{~m}, 2 \mathrm{H}, \mathrm{ArH}) 8.3\left(\mathrm{~s}, 1 \mathrm{H}, \mathrm{H}\right.$ pyr) $8.6(\mathrm{dd}, 1 \mathrm{H}, \mathrm{ArH})$. Anal. Calcd. For $\mathrm{C}_{18} \mathrm{H}_{12} \mathrm{~N}_{4} \mathrm{O}_{2}: \mathrm{C}$, $68.35 ; \mathrm{H}, 3.79 ; \mathrm{N}, 17.72 \%$ found $\mathrm{C}, 68.39 ; \mathrm{H}, 3.82 ; \mathrm{N}, 17.71 \%$.

Compounds $4 \mathrm{~b}-\mathrm{l}$ were similarly prepared the results are tabulated in Table 1.

\section{References:}

1. R.F. Frechette \& M.J.Beach., Synth. Commun., 28(18), 34711998.

2. C.V. Reddy Sastry, K. Srinivasa Rao, V.S.H. Krishnan, K.Rastogi, M.L. Jain, R.K. Varma, R.M. Tripathi, J. Chem 28B, 8821989. 
3. C.B. Chapleo, R.C.M. Butler, D.C. England, P.L. Myen, A.G. Roach, C.F.C. Smith, M.R. Stillings, I.F. Tulloch., J. Med. Chem, 32, 16271989.

4. D.R. Shridhar, K. Srinivasa Rao, A.N. Singh, K. Rastogi, M.L. Jain, S.S. Gardthi, V.S.H. Krishnan, M. Jogibhukta, C.D. Lorekar, H.N. Tripathi \& G.S.T Sai, Indian. J. Chem, 24B, 12631985.

5. K. Varaprasad Rao, PSN Reddy \& V. Sundara Murthy, Indian. J. Chem, 24B, 11201985.

6. D.R. Shridhar, M. Jogibhukta, L.C. Vishwakarma, P.P. Joshi, G.K.A.S.S Narayan, P.P Singh, C. Seshagiri Rao \& A Y. Junnarkar, Indian. J. Chem, 23B, 4451984.

7. C.V. Reddy Sastry, K. Srinivasa Rao, P.P. Singh, C. Seshagiri Rao \& A.Y. Junnarkar, Indian. J. Heterocycl. Chem 1, 1951992.

8. Isao Hayakawa, Tokiyuki Hiramitsu \& Yoshiaki Tanaka, Chem. Pharm. Bull, 32, 49071984.

9. A.R.Kartritzky \& C.W. Rees, Comprehensive Heterocyclic Chemistry vol 5, 291 1984.

10. T.M. Bilodean, R.W.Hangate, W. Randall, R.L. Kendall, R.Rutleelge, A.K. Thomas, R. Robino, EM Raley, W.O 98, 54, 093, Chem. Abstr. 130, ,1999.

11. K. Srinivasa Rao \& C.V. Reddy Sastry., Indian. J. Heterocyclic Chem., 2, 57, 1992.

12. D.R. Shridhar, M. Jogibhukta, V.S.H. Krishnan, Org. Prep. Proced Int, 14(3) 195 1982.

13. A. Costanzo, G. Guerrini, F. Bruni \& S. Severi, J. Heterocycl. Chem. 31, 1369 1994.

14. C.V. Reddy Sastry, K. Srinivasa Rao, V.S.H. Krishnan, K. Rastogi, M.L. Jain, \& G.K.A.S.S Narayan, G.S. Reddy, P.P. Singh, C. Seshagiri Rao \& A.Y. Junnakar, Indian. J. Chem, 29B, 396, 1990.

15. C.V. Reddy Sastry, K. Srinivasa Rao, V.S.H. Krishnan, K. Rastogi \& M.L. Jain, Synthesis 3361988.

16. Representative ${ }^{1} \mathrm{H}$ NMR Spectra: $4 \mathrm{~b}\left(\mathrm{CDCl}_{3}\right): \delta 4.9\left(\mathrm{~s}, 2 \mathrm{H},-\mathrm{OCH}_{2}\right) ; 7.05(\mathrm{~m}, 3 \mathrm{H}$, ArH); 7.7(ABquartet, 4H, $\mathrm{ArH}) ; 8.2(\mathrm{~s}, 1 \mathrm{H}, \mathrm{Hpyr}) ; 8.45(\mathrm{~d}, 1 \mathrm{H}, \mathrm{ArH}) ; 4 \mathrm{~d}\left(\mathrm{CDCl}_{3}\right)$ : $\delta$ 4.9(s, 2H, $\left.\mathrm{OCH}_{2}\right)$ 6.9-7.4(m, 5H, $\left.\mathrm{ArH}\right) ; 7.95(\mathrm{~m}, 2 \mathrm{H}, \mathrm{ArH}) ; 8.2(\mathrm{~s}, \mathrm{H}, \mathrm{Hpyr})$; 8.6(d, $1 \mathrm{H}, \mathrm{ArH}) .4 \mathrm{i}\left(\mathrm{CDCl}_{3}\right): \delta 1.8\left(\mathrm{~d}, 3 \mathrm{H}, \mathrm{CH}_{3}\right) ; 4.9(\mathrm{q}, 1 \mathrm{H}, \mathrm{O}-\mathrm{CH}) ; 7.0-7.6(\mathrm{~m}, 6 \mathrm{H}$, ArH); 8.1(d, 2H, ArH); 8.25(s, 1H, Hpyr); 8.6(d, 1H, ArH).

Received on April 13, 2003. 